\title{
Polymer-Protein Conjugate Particles with Biocatalytic Activity for Stabilization of Water-in-Water Emulsions
}

\author{
Long-Hui Xue, ${ }^{1}$ Chun-Yan Xie, ${ }^{1}$ Shi-Xin Meng, ${ }^{1}$ Rui-Xue Bai, ${ }^{1}$ Xin Yang, ${ }^{1}$ Yaolei Wang, ${ }^{1}$ Shu \\ Wang, ${ }^{1}$ Bernard P. Binks, ${ }^{2}$ Ting Guo ${ }^{1, *}$ and Tao Meng, ${ }^{1, *}$ \\ ${ }^{1}$ School of Life Sciences and Engineering, Southwest Jiaotong University, Chengdu, Sichuan, 610031, P.R. China \\ ${ }^{2}$ School of Mathematics and Physical Sciences, University of Hull, Hull HU6 7RX, UK \\ Keywords: mPEG-protein conjugate particles, Emulsifiers, Water-in-Water emulsions, Biocatalytic activity, Com- \\ partmentalization
}

\begin{abstract}
Water-in-water (w/w) emulsions are attractive micro-compartmentalized platforms due to their outstanding biocompatibility. To address the main disadvantage of poor stability that hampers their practical application, here we report a novel type of polymer-protein conjugate emulsifier obtained by Schiff base synthesis to stabilize w/w emulsions. In particular, the proposed mild approach benefits the modification of proteins of suitable size and wettability as particulate emulsifiers retaining their bioactivity. As demonstrated in a model system, the methoxy polyethylene glycol (mPEG)urease conjugate particles anchor at the w/w interfaces, where they serve as an effective emulsifier-combined-catalyst and catalyze the hydrolysis of urea in water to ammonium carbonate. Our study is unique in that it employs bioactive particles to stabilize w/w emulsions. Considering the characteristics of all-aqueous, compartmental and interfacial biocatalysis of the system, it will open up new possibilities in the life sciences.
\end{abstract}

As a typical compartmentalized system, ${ }^{1}$ emulsions are of particular interest because the numerous droplets can on the one hand confine and enrich cargos in separated microenvironments, ${ }^{2-3}$ while on the other hand facilitate exchange of substances with the continuous phase by interface control when desired.4-5 In addition, their intrinsic properties of high interfacial area ${ }^{6-7}$ and micrometerscale distances of mass transfer make emulsions promising candidates for encapsulation and delivery ${ }^{8-9}$, reaction and separation ${ }^{10-11}$, as well as in the synthesis of functional materials. ${ }^{12-14}$ Despite the extensive success of emulsions in various fields, exploring their biological applications is considerably hampered due to the use of organic solvents as emulsion-forming liquids ${ }^{15}$, which may be harmful to labile cargos such as living cells or proteins or potentially toxic to the spaces they might be introduced. In this context, many efforts have been devoted to develop biocompatible emulsions by the employment of aqueous twophase systems (ATPS). ${ }^{16}$

Water-in-water $(\mathrm{w} / \mathrm{w})$ emulsions are formed when two aqueous solutions consisting of incompatible polymers are homogenized, as long as the composition is above the threshold for phase separation. ${ }^{17}$ The oil-free and allwater nature distinguish $\mathrm{w} / \mathrm{w}$ emulsions with inherent superiority from their counterparts of water-oil emulsions, in compartmentalization of biological molecules, ${ }^{2}$ encap- sulation and delivery of bioactive cargos, ${ }^{18}$ artificial bioreactors, ${ }^{5}$ fibrillosomes ${ }^{19}$ and protocells. ${ }^{1}$ However, significant stabilization of $\mathrm{w} / \mathrm{w}$ emulsions is still a big challenge, due to the ultralow tension of water-water interfaces ${ }^{20-21}$ and the associated large interfacial thickness. ${ }^{22-23}$ In contrast to oil-water emulsions, amphiphilic molecules are not efficient stabilizers for $\mathrm{w} / \mathrm{w}$ emulsions, because of the chemical similarity of the two aqueous phases, and are deemed too small to straddle the thick interfacial zone. ${ }^{24-}$ 25 Recently, colloidal particles have been introduced to enhance the stability of $\mathrm{w} / \mathrm{w}$ emulsions by the so-called Pickering mechanism. ${ }^{26-27}$ The energy of detachment of a spherical particle from a fluid interface $(\Delta G)$ depends on particle radius $(R)$, contact angle $(\theta)$, and interfacial ten$\operatorname{sion}\left(\gamma_{\mathrm{w} / \mathrm{w}}\right),{ }^{28}$

$$
\Delta G=\pi R^{2} \gamma_{w / w}(1-|\cos \theta|)^{2}
$$

Strong adsorption of larger particles to the interface can dramatically increase the detachment energy, ${ }^{29-30}$ despite the low interfacial tension, ${ }^{31}$ only if the diameter of the particles exceeds several hundred nm..5, ${ }^{22}$ Moreover, the coverage of particles at the surface of emulsion droplets serves as a steric barrier against the coalescence of the droplets, therefore significantly slowing down macroscopic phase separation..$^{32}$ Over the past years, by delicate control over the size or geometry of the particles, reason- 
ably stable w/w emulsions have been prepared using for example latex particles, ${ }^{28}$ gibbsite nanoplates ${ }^{23}$ and cellulose nanorods. ${ }^{3}$ Considering the promising potential of $\mathrm{w} / \mathrm{w}$ emulsions in biological applications, protein- or polysaccharide-based emulsifiers are particularly attractive. In the early studies, liposomes, ${ }^{5}$ protein nanofibrils, ${ }^{19}$ polysaccharide-coated protein particles ${ }^{34}$ and heat-denatured protein particles ${ }^{32,34-35}$ have been designed.

Conjugating protein molecules with polyethylene glycol (PEG), a well-established technique known as PEGylation, can efficiently improve the stability and potency of protein pharmaceuticals. ${ }^{36}$ PEGs are non-toxic, nonimmunogenic, non-antigenic, water-soluble polymers and clinically approved. ${ }^{37}$ It is believed that PEG chains, grafted on protein by covalent bonds, entangle around the protein surface through hydrophobic interaction ${ }^{38}$ and such conjugation increases the size and molecular weight of the conjugated protein (See Table $\mathrm{S}$ ). Moreover, proteins most readily aggregate under non-denaturing conditions at $\mathrm{pH}$ close to the isoelectric point (PI).39 This a feasible and gentle method to tailor a suitable size $\mathrm{4}^{\mathrm{O}}$ and wettability ${ }^{4-42}$ of protein conjugates. Inspired by this, here we propose a novel highly biocompatible methoxy PEG (mPEG)-protein conjugate as the stabilizer of $w / w$ emulsions, which are readily achieved by reacting a protein (Bovine Serum Albumin, BSA) with mPEG-acetaldehyde (ALD) via Schiff base chemistry at a $\mathrm{pH}$ near the PI of the protein (Figure 1a). Depending on the reaction duration, the diameter of the conjugates can be easily controlled to a preferred range of between 200 and $500 \mathrm{~nm}$ (PEGylation-induced gradual aggregation of BSA to form conjugate particles). Furthermore, in a widely used PEG/dextran (Dex) system which phase separates into two aqueous phases, native BSA molecules readily partition into the Dex-rich phase (no stable emulsion) whilst the mPEG-BSA conjugate particles display interfacial affinity and hence act to stabilize $\mathrm{w} / \mathrm{w}$ emulsions. More importantly, mPEG has good solubility and biocompatibility for bioactive protein 43 and the bioactivity of the protein is preserved. Using urease as a model protein, mPEG-urease conjugate particles are preferentially situated at the water-water interfaces where they play a dual role not only as emulsion stabilizers but also as interfacial biocatalysts.
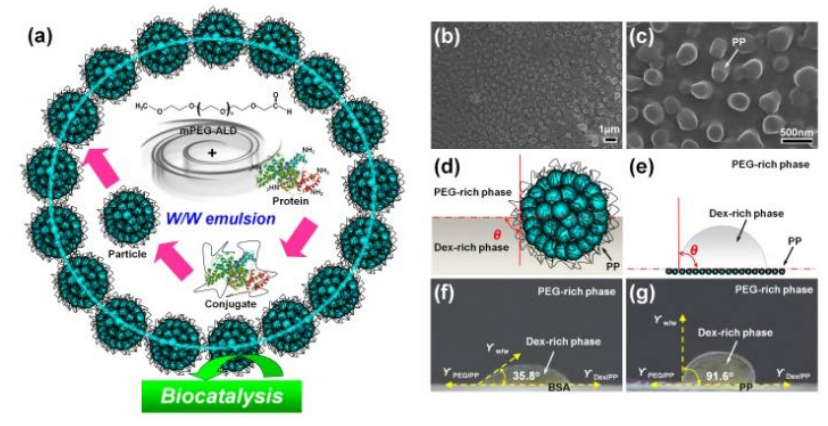

Figure 1. Design of the polymer-protein conjugate particles with biocatalytic activity for the stablizition of water-inwater emulsions (a). Morphology and wettability of mPEGBSA conjugate particles. The SEM images $\times 5000(b), \times 30000$ (c), scheme of a conjugate particle at the W/W interface (d) and measurement of conjugate particle surface wettability (e), three-phase contact angle $(\theta)$ photographs of BSA (f) and mPEG-BSA conjugate particles, PP (g).

The size and surface wettability of particles are thought to be significant in stabilizing $\mathrm{w} / \mathrm{w}$ Pickering emulsions. In order to increase the size and tailor the wettability of BSA, mPEG-ALD is grafted to BSA by a Schiff base reaction (see Figure $S_{2}$ and Figure $S_{3}$ ). To investigate the morphology of the mPEG-BSA conjugate particles, SEM images are shown in Figure $1 \mathrm{~b}$ and $1 \mathrm{c}$. It can be seen that mPEG-BSA conjugate particles have diameters in the range $200-500 \mathrm{~nm}$. Compared with BSA molecules (4 $\mathrm{nm} \times 4 \mathrm{~nm} \times 14 \mathrm{~nm})^{44}$ the irregular spherical aggregates indicate that the size of $\mathrm{mPEG}-\mathrm{BSA}$ conjugate particles is larger than the native BSA molecules due to the aggregation of many mPEG-BSA molecules.

The three-phase contact angle $\theta$ of a particle stabilizer at the water-water interface is a fundamental parameter. When $\theta$ approaches $90^{\circ}$ the emulsifiers prefer to anchor at the interface which is beneficial for the stability of $w / w$ Pickering emulsions (Figure $1 \mathrm{~d}$ ). As shown in Figure 1e, $\theta$ is determined by depositing a particle film on a glass surface (Figure $\mathrm{S}_{1}$ ). The value of $\theta$ of $35.8^{\circ}$ is displayed for native BSA film (Figure if) implying a marked preference of the BSA molecules for the Dex-rich phase. In contrast, $\theta$ for mPEG-BSA conjugate particles increases to $91.6^{\circ}$ (Figure $1 \mathrm{~g}$ ), which is a suitable wettability to assemble these particles at the interface to stabilize w/w emulsions. This fact verifies that mPEG-ALD modification can efficiently increase the hydrophobicity of BSA and strengthen the affinity of BSA to PEG due to the long $\left(-\mathrm{CH}_{2} \mathrm{CH}_{2} \mathrm{O}-\right.$ )$_{n}$ segments grafted on the protein.

To prove the stabilizing effect of $\mathrm{mPEG}$-BSA conjugate particles, the storage stability of the $\mathrm{w} / \mathrm{w}$ emulsions at the natural $\mathrm{pH} 7.0$ after $24 \mathrm{~h}$ is examined and compared with those without any emulsifier or using native BSA as emulsifier. The diameter of mPEG-BSA conjugate particles is about $300 \mathrm{~nm}$ at $\mathrm{pH} 7.0$ (Figure $\mathrm{S}_{4}$ ). Figure 2a shows the macroscopic appearance of the mixtures. It can be seen that the blank ATPS completely phase separates (within $30 \mathrm{~min}$ ) to a Dex-rich phase at the bottom and a PEG-rich phase at the top. Addition of native BSA has no effect on the emulsification tendency. However, the addition of mPEG-BSA conjugate particles led to stabilization of Dexin-PEG emulsions, i.e. the dextran droplets do not fuse even though they sediment. A fluorescence microscopy image recorded a few minutes after preparation of the emulsion in the presence of $0.5 \mathrm{wt} \%$ mPEG-BSA conjugate particles shows the labeled particles situated at the water-water interface of droplets seen in Figure $2 \mathrm{~b}$. The result is due to the large size and suitable wettability of mPEG-BSA conjugate particles. The emulsion remains stable to coalescence for over a week with no change in the drop size distribution. 

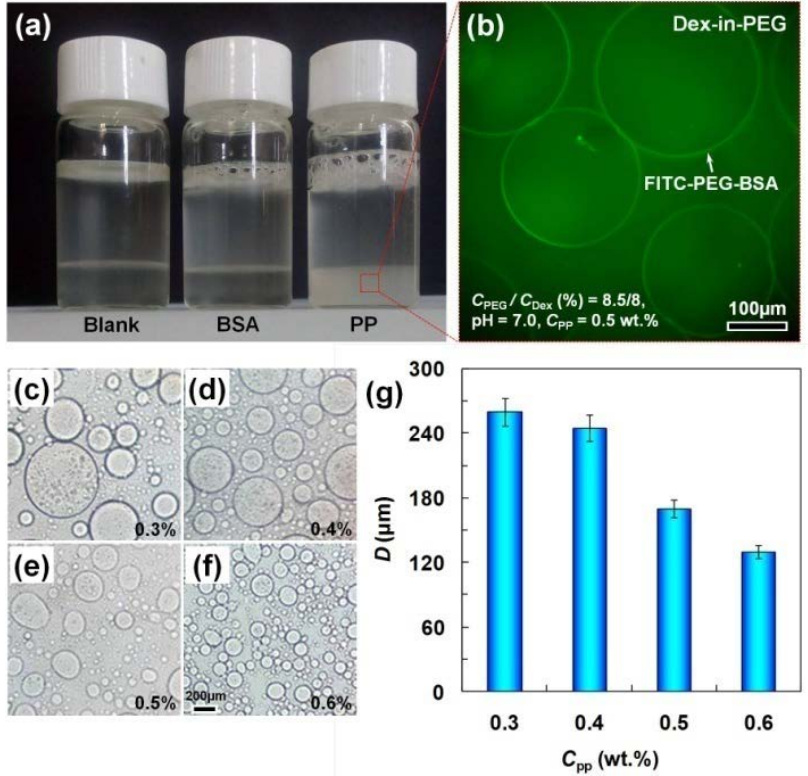

Figure 2. (a) Digital photographs of $\mathrm{w} / \mathrm{w}$ emulsions at $\mathrm{pH} 7.0$ containing either no emulsifier (blank), 0.5 wt.\% BSA or 0.5 wt.\% mPEG-BSA conjugate particles after $24 \mathrm{hr}$ storage at room temperature, (b) fluorescence microscopy image of Dex-in-PEG emulsion in the presence of $0.5 \mathrm{wt} . \% \mathrm{mPEG}-\mathrm{BSA}$ conjugate particles and $20 \mathrm{ppm}$ mPEG-BSA-FITC conjugate particles (label). Optical microscopy images of Dex-in-PEG emulsion droplets at $\mathrm{pH} 7.0$ at various concentrations of mPEG-BSA conjugate particles, $C_{\mathrm{PP}}(\mathrm{wt} . \%)$ : (c) 0.3 , (d) 0.4 , (e) 0.5 , (f) o.6. (g) Effect of $C_{P P}$ on the mean diameter of the emulsion droplets. The emulsions contain $8.5 \mathrm{wt} \%$ PEG and 8 wt.\% Dex with the initial volume fraction of Dex phase being 0.3 .

To learn more about the droplet size for w/w emulsions, we measured the average droplet size as a function of mPEG-BSA conjugate particle concentration. The droplet size for a given polymer mixture composition is inversely correlated with the concentration of mPEG-BSA conjugate particles (Figure $2 \mathrm{c}-\mathrm{f}$ ). The droplet size increases as the particle concentration is reduced (Figure 2g), which can be explained by the fact that incompletely covered small droplets will coalesce yielding larger droplets until the interface is fully stabilized. ${ }^{45-46}$ Alongside this, the stability to coalescence of the emulsions improved at higher particle concentration.

To explore the effect of composition on the stability of water-in-water emulsions, the evolution with time of emulsions at different compositions in the presence of 0.5 wt.\% mPEG-BSA conjugate particles is shown in Figure 3. The $\mathrm{w} / \mathrm{w}$ emulsions are stable to coalescence for at least a week at all compositions. The fluorescence images of $w / w$ emulsions at different compositions show that dextran droplets are formed in a continuous PEG phase for $C_{\mathrm{PEG}}>$ 4 wt.\% (Figure 3e), while PEG droplets are formed in a continuous dextran phase at lower PEG concentrations (Figure $3 \mathrm{f}$ ). Emulsion phase inversion takes place when the volume fraction $(\phi)$ of the dextran phase becomes larger than about 0.45 due to the packing of droplets ${ }^{47}$. In both cases, the droplets are covered with a layer of
mPEG-BSA conjugate particles (Figure $3 \mathrm{~g}$ and $\mathrm{h}$ ). The bulky mPEG-BSA conjugate particles render the droplets heavy which causes them to settle to the vial bottom. The relatively rapid sedimentation of dextran droplets compared to PEG droplets is due to the large difference in the molar masses of the two polymers. The nominal weightaverage molar mass is $500 \mathrm{kDa}$ for dextran but only $8 \mathrm{kDa}$ for PEG. Hence the lighter PEG droplets have relatively slower speed during the process of sedimentation. These results suggest that the sedimentation velocity of $\mathrm{w} / \mathrm{w}$ emulsions can be controlled by choosing suitable molar masses of mPEG-protein conjugate particles, PEG and dextran. In addition, $\mathrm{pH}$ is an interesting variable affecting the stability of Pickering emulsions stabilized by polymer-protein conjugate particles linked to changes in the particle charge (Figures $\mathrm{S}_{5}-\mathrm{S}_{7}$ ).

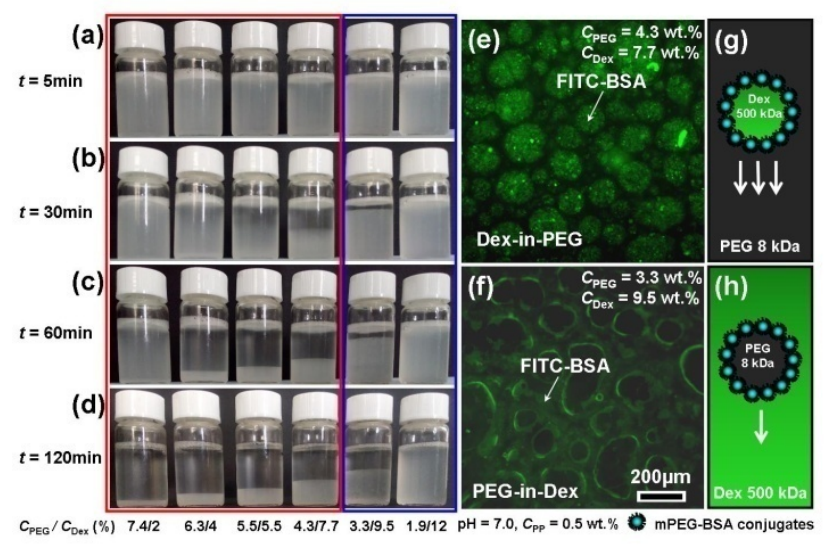

Figure 3. (a-d) Evolution with time of emulsions formed by PEG-Dex mixtures of different composition in the presence of 0.5 wt.\% mPEG-BSA conjugate particles at $\mathrm{pH}$ 7.o. $C_{\mathrm{PEG}} / C_{\mathrm{Dex}}(\mathrm{wt} . \%)$ from left to right: $7.4 / 2 ; 6.3 / 4 ; 5.5 / 5.5$; $4.3 / 7.7 ; 3.3 / 9.5$ and $1.9 / 12$. Those enclosed by the red box are Dex-in-PEG whilst those in the blue box are PEG-in-Dex. Fluorescence micrographs of the FITC-BSA signal for Dex phase in the presence of $0.5 \mathrm{wt} . \%$ mPEG-BSA conjugate particles with different compositions, (e) $C_{\mathrm{PEG}}=4.3 \mathrm{wt} . \%, C_{\mathrm{Dex}}=$ 7.7 wt. $\%$ and (f) $C_{\text {PEG }}=3.3$ wt. $\%, C_{\text {Dex }}=9.5$ wt.\%. $(\mathrm{g}, \mathrm{h})$ Sketch of Dex-in-PEG and PEG-in-Dex drops respectively with the former sedimenting more rapidly.

Urease can catalyze the hydrolysis of urea to ammonium carbonate. As shown in Figure 4, a comparison of the catalytic activity of particle-stabilized emulsions is demonstrated. In one case the enzyme is incorporated within the particles (mPEG-urease conjugate particles) and in the other case it is dissolved in the dextran droplets. Urease prefers to be partitioned in the Dex-rich phase (see Table S2). Figure 4a shows the evolution of the product conductivity in the bottom phase (emulsion) with time for Dex-in-PEG emulsions stabilized by either mPEG-urease conjugate particles (squares) or heatdenatured $\mathrm{mPEG}$-urease conjugate particles with urease dissolved in the droplets (circles). The amount of product in the first case is higher than in the second case. In both cases the emulsions are stable to coalescence up to at least 90 min (insert). Figure $4 \mathrm{~b}$ and Figure $4 \mathrm{c}$ are the schematic illustration of the enzyme reactions. Comparing Figure $4 \mathrm{~b}$ and Figure $4 \mathrm{c}$, the only difference is that the 
native urease is mainly in the dextran droplets (Figure $4 \mathrm{~b}$ ) whilst the bioactive $\mathrm{mPEG}$-urease conjugate particles are mainly at the $\mathrm{w} / \mathrm{w}$ emulsion interfaces (Figure $4 \mathrm{c}$ ). The particle-stabilized emulsions with the enzyme incorporated in the particles enable an increased possibility of contact between enzyme and substrate, and reduce the diffusion distance of the substrate from the continuous phase to the droplet phase. Therefore, the catalytic activity is higher when the enzyme is incorporated in the particles than when it is dissolved in dextran droplets. Consequently, the mPEG-protein conjugate particles prepared by Schiff base reaction in this work not only exhibit bioactivity but also enhance enzyme reactions through locating at the interface of $w / w$ emulsions.

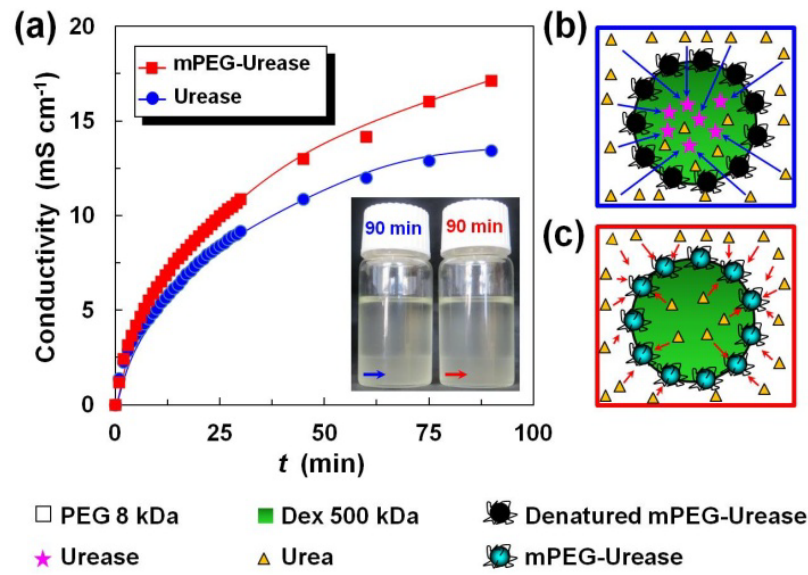

Figure 4. Biocatalytic activity of mPEG-urease conjugate particles. (a) Evolution of the product conductivity with time for Dex-in-PEG emulsions (bottom phase). Squares - stabilised by $\mathrm{mPEG}$-urease conjugate particles; circles - represent urease dissolved in Dex droplets stabilized by heatdenatured mPEG-urease conjugate particles. Inset - photos of corresponding vessels after 90 min. Schematic illustration of the reaction with urease in Dex droplet (b) and with mPEG-urease particles at w/w droplet interface (c). Emulsions contain 8.5 wt.\% PEG, 8 wt.\% dextran, $20 \mathrm{mg} \mathrm{mL}^{-1}$ urea, $\mathrm{pH}=7 \cdot 0$.

In summary, we report the preparation of bioactive particulate emulsifiers prepared by conjugating protein with mPEG to efficiently stabilize w/w emulsions. In comparison with native BSA which readily partitions into the Dex-rich phase, the mPEG-BSA conjugate emulsifiers developed here adsorb strongly at the water-water interface due to their suitable size (200-500 $\mathrm{nm})$ and wettability $(\theta$ $\left.=91 \cdot 6^{\circ}\right)$. Both Dex-in-PEG and PEG-in-Dex emulsions can be readily obtained by varying the initial volume fraction of the two phases. The size of the resulting emulsion droplets decreases upon increasing the particle concentration. Considering the properties of all-aqueous, compartmental and interfacial biocatalyst particle-stabilized $\mathrm{w} / \mathrm{w}$ emulsions, we expect them to have wide applications in life sciences, such as in biomedical applications and biomimetic microreactors.

\section{ASSOCIATED CONTENT}

\section{Supporting Information}

Materials, methods, techniques and supplementary data (PDF). The Supporting Information is available free of charge on the ACS Publications website at DOI: 10.1021/acsmacrolett.XXXXXXX.

\section{AUTHOR INFORMATION}

\section{Corresponding Author \\ *E-mail: tt-g-19@163.com, taomeng@swjtu.edu.cn. Notes}

The authors declare no competing financial interest.

\section{ACKNOWLEDGMENT}

This work was supported by the National Natural Science Foundation of China $(21106115,21406181)$, the Fundamental Research Funds for the Central Universities (SWJTU12CX049, 2682015CXo5o) and the Science and Technology Department Foundation of Sichuan Province (2017GZo411). The authors gratefully acknowledge the help of Dr. Feng Yang of the Superconductivity and New Energy R\&D Center at Southwest Jiaotong University for the SEM micrographs, Dr. Ming-yu Wu of the School of Life Sciences and Engineering at Southwest Jiaotong University for NMR analysis and Prof. D. Lu of the Department of Chemical Engineering at Tsinghua University for discussions.

\section{ABBREVIATIONS}

w/w, water-in-water; mPEG, methoxy polyethylene glycol; ATPS, aqueous two-phase systems; PEG, polyethylene glycol; Dex, dextran; BSA, Bovine Serum Albumin; PI, isoelectric point; PP, polymer-protein conjugate particles; FITC, fluorescein isothiocyanate.

\section{REFERENCES}

(1) Keating, C. D., Aqueous Phase Separation as a Possible Route to Compartmentalization of Biological Molecules. Acc. Chem. Res. 2012, 45, 2114-2124.

(2) Strulson, C. A.; Molden, R. C.; Keating, C. D.; Bevilacqua, P. C., RNA Catalysis through Compartmentalization. Nat. Chem. 2012, 4, 941-946.

(3) Huang, X.; Li, M.; Green, D. C.; Williams, D. S.; Patil, A. J.; Mann, S., Interfacial Assembly of Protein-Polymer NanoConjugates into Stimulus-Responsive Biomimetic Protocells. Nat. Commun. 2013, 4, 2239-2247.

(4) Yang, H.; Fu, L.; Wei, L.; Liang, J.; Binks, B. P., Compartmentalization of Incompatible Reagents within Pickering Emulsion Droplets for One-Pot Cascade Reactions. J. Am. Chem. Soc. 2015, 137, 1362-1371.

(5) Dewey, D. C.; Strulson, C. A.; Cacace, D. N.; Bevilacqua, P. C.; Keating, C. D., Bioreactor Droplets from Liposome-Stabilized All-Aqueous Emulsions. Nat. Commun. 2o14, 5, 4670-4678.

(6) Crossley, S.; Faria, J.; Shen, M.; Resasco, D. E., Solid Nanoparticles that Catalyze Biofuel Upgrade Reactions at the Water/Oil Interface. Science 2010, 327, 68-72.

(7) Wiese, S.; Spiess, A. C.; Richtering, W., Microgel-Stabilized Smart Emulsions for Biocatalysis. Angew. Chem. Int. Ed. 2013, 52, 576-579.

(8) Bai, R. X.; Xue, L. H.; Dou, R. K.; Meng, S. X.; Xie, C. Y.; Zhang, Q.; Guo, T.; Meng, T., Light-Triggered Release from Pickering Emulsions Stabilized by $\mathrm{TiO}_{2}$ Nanoparticles with Tailored Wettability. Langmuir 2016, 32, 9254-9264.

(9) McClements, D. J.; Decker, E. A.; Weiss, J., Emulsion-Based Delivery Systems for Lipophilioc Bioactive Components. J. Food Sci. 2007, 72, R109-R124. 
(10) Yang, H. Q.; Zhou, T.; Zhang, W. J., A Strategy for Separating and Recycling Solid Catalysts Based on the $\mathrm{pH}-$ Triggered Pickering-Emulsion Inversion. Angew. Chem. Int. Ed. 2013, 52, 7455-7459.

(11) Chen, Z. W.; Zhou, L.; Bing, W.; Zhang, Z. J.; Li, Z. H.; Ren, J. S.; Qu, X. G., Light Controlled Reversible Inversion of Nanophosphor-Stabilized Pickering Emulsions for Biphasic Enantioselective Biocatalysis. J. Am. Chem. Soc. 2014, 136, 74987504 .

(12) Walther, A.; Hoffmann, M.; Mueller, A. H. E., Emulsion Polymerization using Janus Particles as Stabilizers. Angew. Chem. Int. Ed. 2008, 47, 711-714.

(13) Kimmins, S. D.; Cameron, N. R., Functional Porous Polymers by Emulsion Templating: Recent Advances. Adv. Funct. Mater. 2011, 21, 211-225.

(14) Imhof, A.; Pine, D. J., Ordered Macroporous Materials by Emulsion Templating. Nature 1997, 389, 948-951.

(15) Zhang, L.; Cai, L.-H.; Lienemann, P. S.; Rossow, T.; Polenz, I.; Vallmajo-Martin, Q.; Ehrbar, M.; Na, H.; Mooney, D. J.; Weitz, D. A., One-Step Microfluidic Fabrication of Polyelectrolyte Microcapsules in Aqueous Conditions for Protein Release. Angew. Chem. Int. Ed. 2016, 55, 13470-13474.

(16) Esquena, J., Water-in-Water (W/W) Emulsions. Curr. Opin. Colloid Interface Sci. 2016, 25, 109-119.

(17) Frith, W. J., Mixed Biopolymer Aqueous Solutions-Phase Behaviour and Rheology. Adv. Colloid Interface Sci. 2010, 161, 486 o.

(18) Hann, S. D.; Niepa, T. H. R.; Stebe, K. J.; Lee, D., One-Step Generation of Cell-Encapsulating Compartments via Polyelectrolyte Complexation in an Aqueous Two Phase System. ACS Appl. Mater. Interfaces 2016, 8, 25603-25611.

(19) Song, Y.; Shimanovich, U.; Michaels, T. C. T.; Ma, Q. M.; Li, J. M.; Knowles, T. P. J.; Shum, H. C., Fabrication of Fibrillosomes from Droplets Stabilized by Protein Nanofibrils at All-Aqueous Interfaces. Nat. Commun. 2016, 7, 12934-12941.

(20) Atefi, E.; Mann, J. A.; Tavana, H., Ultralow Interfacial Tensions of Aqueous Two-Phase Systems Measured Using Drop Shape. Langmuir 2014, 30, 9691-9699.

(21) Ryden, J.; Albertsson, P., Interfacial Tension of DextranPolyethylene Glycol-Water Two-Phase Systems. J. Colloid Interface Sci. 1971, 37, 219-222.

(22) Tromp, R. H.; Vis, M.; Erne, B. H.; Blokhuis, E. M., Composition, Concentration and Charge Profiles of WaterWater Interfaces. J. Phys. Condens. Matter 2o14, 26, 464101.

(23) Vis, M.; Opdam, J.; van 't Oor, I. S. J.; Soligno, G.; van Roij, R.; Tromp, R. H.; Erne, B. H., Water-in-Water Emulsions Stabilized by Nanoplates. Acs Macro Lett. 2015, 4, 965-968.

(24) Zhang, Y. L.; Wu, F.; Yuan, W. E.; Jin, T., Polymersomes of Asymmetric Bilayer Membrane Formed by Phase-Guided Assembly. J. Control. Release 2010, 147, 413-419.

(25) Buzza, D. M. A.; Fletcher, P. D. I.; Georgiou, T. K.; Ghasdian, N., Water-in-Water Emulsions Based on Incompatible Polymers and Stabilized by Triblock Copolymers-Templated Polymersomes. Langmuir 2013, 29, 14804-14814.

(26) Binks, B. P., Particles as Surfactants - Similarities and Differences. Curr. Opin. Colloid Interface Sci. 2002, 7, 21-41.

(27) Binks, B. P., Colloidal Particles at Liquid Interfaces. Phys. Chem. Chem. Phys. 2007, 9, 6298-6299.

(28) Balakrishnan, G.; Nicolai, T.; Benyahia, L.; Durand, D., Particles Trapped at the Droplet Interface in Water-in-Water Emulsions. Langmuir 2012, 28, 5921-5926.

(29) Pickering, S. U., Emulsions. J. Chem. Soc., Trans. 1907, 91, 2001-2021.

(30) Binks, B. P.; Horozov, T. S., Colloidal Particles at Liquid Interfaces. Cambridge University Press: Cambridge, 2006.

(31) Liu, Y.; Lipowsky, R.; Dimova, R., Concentration Dependence of the Interfacial Tension for Aqueous Two-Phase
Polymer Solutions of Dextran and Polyethylene Glycol. Langmuir 2012, 28, 3831-3839.

(32) Nguyen, B. T.; Nicolai, T.; Benyahia, L., Stabilization of Water-in-Water Emulsions by Addition of Protein Particles. Langmuir 2013, 29, 10658-10664.

(33) Peddireddy, K. R.; Nicolai, T.; Benyahia, L.; Capron, I., Stabilization of Water-in-Water Emulsions by Nanorods. ACS Macro Lett. 2016, 5, 283-286.

(34) de Freitas, R. A.; Nicolai, T.; Chassenieux, C.; Benyahia, L., Stabilization of Water-in-Water Emulsions by PolysaccharideCoated Protein Particles. Langmuir 2016, 32, 1227-1232.

(35) Gonzalez-Jordan, A.; Nicolai, T.; Benyahia, L., Influence of the Protein Particle Morphology and Partitioning on the Behavior of Particle-Stabilized Water-in-Water Emulsions. Langmuir 2016, 32, 7189-7197.

(36) Webber, M. J.; Appel, E. A.; Vinciguerra, B.; Cortinas, A. B.; Thapa, L. S.; Jhunjhunwala, S.; Isaacs, L.; Langer, R.; Anderson, D. G., Supramolecular PEGylation of Biopharmaceuticals. Proc. Natl. Acad. Sci. U. S. A. 2016, 113, 14189-14194.

(37) Harris, J. M.; Chess, R. B., Effect of Pegylation on Pharmaceuticals. Nat. Rev. Drug Discov. 2003, 2, 214-221.

(38) Yang, C.; Lu, D. N.; Liu, Z., How PEGylation Enhances the Stability and Potency of Insulin: A Molecular Dynamics Simulation. Biochemistry 2011, 50, 2585-2593.

(39) Majhi, P. R.; Ganta, R. R.; Vanam, R. P.; Seyrek, E.; Giger, K.; Dubin, P. L., Electrostatically Driven Protein Aggregation: $\beta$ Lactoglobulin at Low Ionic Strength. Langmuir 2006, 22, 91509159 .

(40) Zhao, T.; Yang, Y.; Zong, A. Z.; Tan, H. N.; Song, X. L.; Meng, S. O.; Song, C. X.; Pang, G. L.; Wang, F. S., N-Terminal PEGylation of Human Serum Albumin and Investigation of Its Pharmacokinetics and Pulmonary Microvascular Retention. Biosci. Trends 2012, 6, 81-88.

(41) Harris, J. M.; Sedaghat-Herati, M. R., Preparation and Use of Polyethylene Glycol Propionaldehyde. U.S. Patent 5,252,714, 1993. (42) Franco, T. T.; Andrews, A. T.; Asenjo, J. A., Use of Chemically Modified Proteins to Study the Effect of A Single Protein Property on Partitioning in Aqueous Two-Phase Systems: Effect of Surface Hydrophobicity. Biotechnol. Bioeng. 1996, 49, 300-308.

(43) Zalipsky, S., Chemistry Of Polyethylene-Glycol Conjugates with Biologically-Active Molecules. Adv. Drug Del. Rev. 1995, 16, 157-182.

(44) McClellan, S. J.; Franses, E. I., Effect of Concentration and Denaturation on Adsorption and Surface Tension of Bovine Serum Albumin. Colloids Surf. B. Biointerfaces 2003, 28, 63-75. (45) Arditty, S.; Whitby, C. P.; Binks, B. P.; Schmitt, V.; LealCalderon, F., Some General Features of Limited Coalescence in Solid-Stabilized Emulsions. Eur. Phys. J. E 2003, 11, 273-281.

(46) Nallamilli, T.; Mani, E.; Basavaraj, M. G., A Model for the Prediction of Droplet Size in Pickering Emulsions Stabilized by Oppositely Charged Particles. Langmuir 2014, 30, 9336-9345.

(47) Becher, P., Emulsion: Theory and Practice. Reinhold: New York, 1965 . 
SYNOPSIS TOC

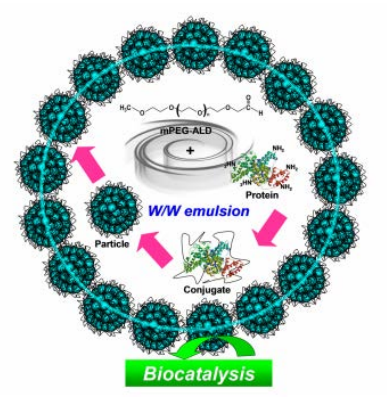

For Table of Contents use only $\left(4 \mathrm{~cm}^{*} 4 \mathrm{~cm}\right)$

Polymer-Protein Conjugate Particles with Biocatalytic Activity for Stabilization of Water-in-Water Emulsions

Long-Hui Xue, ${ }^{1}$ Chun-Yan Xie, ${ }^{1}$ Shi-Xin Meng, ${ }^{1}$ Rui-Xue Bai, ${ }^{1}$ Xin Yang, ${ }^{1}$ Yaolei Wang,, Shu Wang, ${ }^{1}$ Bernard P. Binks, ${ }^{2}$ Ting Guo ${ }^{1, *}$ and Tao Meng ${ }^{1, *}$

6 